\title{
Changes in Iron Status of Hypertensive Patients
}

\author{
Obeagu Emmanuel Ifeanyi \\ Department of Medical Laboratory Science, Imo State University, Owerri, Nigeria. \\ *Corresponding Author: Obeagu Emmanuel Ifeanyi, Department of Medical Laboratory Science, \\ Imo State University, Owerri, Nigeria.
}

\begin{abstract}
Iron is a key player in hemoglobin synthesis and erythrocyte production. Iron levels can change before and after menopause, in patients with insulin resistant hypertension. Studies have shown that iron deficiency may contribute to the pathogenesis of pulmonary hypertension. Different search databases were utilized in this review such as Pubmed, Google Scholar, Researchgate, Academia Edu and so many others. Therefore, its metabolism in mammalians its metabolism in mammalians is very complex and stringently controlled by many different genes and proteins. Restoring iron levels improves quality of life as it is an essential nutrient for survival.
\end{abstract}

Keywords: iron status, high blood pressure, iron metabolism

\section{INTRODUCTION}

Iron is the principal catalyst that allows for oxygen utilization. The electronic structure of ground state molecular oxygen provides inherent stability (two unpaired electrons with parallel spin); so called spin restriction. Ground state molecular oxygen is, therefore, a relatively unreactive molecule. In order to facilitate oxygen utilization for metabolism, conversion to a reactive state (activation) is achieved via single electron transfer reactions. Iron, as a classical transition metal, has the ability to exist in different states of valence and, therefore, the ability to donate or accept electrons singly, enabling it to convert oxygen to a reactive and therefore metabolically active state. Consequently, body iron requirements are almost exclusively involved with some aspect of oxygen utilization. Notable examples include: respiration, molecular transport, molecular storage, antioxidant protection and biosynthesis (Lathaet al., 2018).

Arterial hypertension is a major cause of morbidity and mortality because of its association with coronary heart disease, cerebrovascular disease and renal disease. The extent of target organ involvement (i.e. heart, brain and kidneys) determines outcome. North American studies have shown that hypertension is a major contributor to 500000 strokes (250000 deaths) and 1000000 myocardial infarctions (500000 deaths) per annum (Foe" $\mathrm{x}$ and Sear, 2015).Over the past decade the management of hypertension has changed with the recognition that there is no threshold below which elevated blood pressure causes no threat to health. Recent guidelines, including those of the British Hypertension Society, make it clear that treatment of isolated systolic hypertension is as important as that of systolic and diastolic hypertension. The threshold above which hypertension should be treated to prevent longterm complications is now $140 / 90 \mathrm{~mm} \mathrm{Hg}$. Indeed, in Stage 1 hypertension, treatment of isolated systolic hypertension (systolic 140- $159 \mathrm{~mm} \mathrm{Hg}$, diastolic $<90 \mathrm{~mm} \mathrm{Hg}$ ), reduces the prevalence of left ventricular hypertrophy, a predictor of future morbidity and mortality. There is also a $42 \%$ reduction of the risk of stroke and a reduction in the risk of dementia. The hypertension optimal treatment (HOT) study indicates that the treatment goal is to reduce blood pressure to $140 / 85 \mathrm{~mm} \mathrm{Hg}$. It is also established that high normal blood pressure $(130-139 / 85-89 \mathrm{~mm} \mathrm{Hg})$ progresses to Stage 1 hypertension (>140/>90 mm $\mathrm{Hg}$ ) in $>37 \%$ of individuals $<64 \mathrm{yr}$ and $>49 \%$ of those $>65 \mathrm{yr}$ (Foe"x and Sear, 2015).

Iron plays a critical role in oxygen transport and iron depletion affects regulation of hypoxiainducible factors. In a preclinical study, a reduction in iron and ferritin levels resulted in marked pulmonary vascular remodelling that was reversed by iron supplementation (Cotroneoet al., 2015). Similarly, studies in humans have noted that iron augmentation or depletion differentially affect pulmonary 
vascular responses to hypoxia. Iron deficiency is highly prevalent in cohorts with idiopathic pulmonary arterial hypertension (PAH) (Rhodes et al., 2011). Moreover, in patients with PAH and iron deficiency, iron augmentation increased exercise endurance time and aerobic capacity. These preclinical and epidemiological studies suggest that iron may be an important factor in the regulation of pulmonary hemodynamic and that iron deficiency may contribute to the pathogenesis of pulmonary hypertension (Matthew et al., 2016). Iron deficiency is defined as reduced iron availability due to depleted iron stores (e.g. Pregnancy), inadequate dietary iron intake (e.g., malnutrition), repressed iron absorption (e.g., inflammatory setting), or iron loss (e.g., menstrual blood loss in women of reproductive age). It has a significant negative impact on the quality of life. It affects intrauterine development, reduces physical and mental exercise capacity, and increases morbidity when concurrent with cardiovascular diseases, such as chronic heart failure(CHF), acute heart failure(AHF),pulmonary arterial hypertension $(\mathrm{PAH})$, chronic obstructive pulmonary disease (COPD), and abdominal aortic aneurysm (AAA) (Nickolet al., 2015). The aetiology of iron deficiency in cardiovascular diseases is not entirely clear, but evidence supports the role of inflammation, and particularly of the inflammatory cytokine interleukin-6 in blocking iron absorption in the gut.The interplay between iron and oxygen is longstanding and central to all aerobic life. Tight regulation of these interactions including homeostatic regulation of iron utilization ensures safe usage of this limited resource. However, when control is lost adverse events can ensue, which are known to contribute to an array of disease processes. Recently, associations between disrupted iron homeostasis and pulmonary artery hypertension $(\mathrm{PAH})$ have been described with the suggestion that there is a contributory link with disease (Lathaet al., 2018).

Iron deficiency is frequently encountered in chronic left heart failure (Ruiteret al., 2011). The cause of iron depletion is thought to be inflammation mediated and/or due to renal failure. Both conditions cause an increase in hepcidin concentration (increased production in the liver or reduced breakdown by the kidney), which causes a down regulation of iron release from storage sites and decreased uptake of iron from the gut mucosa (Ruiteret al., 2011). It has also been shown that restoring iron levels improves quality of life and New York Health
Association (NYHA) functional class in irondeficient patients with chronic left heart failure. In contrast to iron deficiency in left ventricular failure, no clinical data exist for right ventricular failure, although (Ruiteret al., 2011) reported that anaemia in pulmonary hypertension decreased survival. Pulmonary hypertension is the most common cause of right ventricular failure. Since iron is important in increasing oxidative metabolism of the hypertrophied right ventricle to cope with the increased afterload and for skeletal muscles to perform physical exercise, iron deficiency might thus have detrimental effects in pulmonary hypertension. However, data are lacking about the prevalence and effect of iron deficiency in right heart failure (Ruiteret al., 2011).

In CKD group, iron metabolism is altered compared to controls. Here, serum iron levels are low due to inadequate secretion of erythropoietin, erythropoiesis is halted leading to decreased $\mathrm{Hb}$ and serum iron. Serum ferritin stores are not utilized due to associated inflammation giving rise to near normal ferritin levels (Veena and Astagimath, 2019). The relationship between serum ferritin and hypertension has not been well established in women and has been controversial, but reports have found an association between serum ferritin and hypertension in men (Dong-Hoonet al., 2012).

\section{IRON METABOLISM}

Iron is an essential nutrient for survival of most living organisms and a component or co-factor of hundreds of proteins and enzymes. The biological importance of iron is widely attributed to its chemical properties. There are two oxidative forms, ferrous $(\mathrm{Fe}+2)$ and ferric $(\mathrm{Fe}+3)$ iron, and iron is able to accept and donate electrons, being useful in the catalytic center of fundamental biochemical reactions. In mammals, these reactions are performed by iron-containing proteins, iron-sulphur enzymes, hemeproteins and iron-containing enzymes (non-heme and non-iron-sulphur enzymes). The activity of most of these enzymes decreases in tissue iron deficiency (Ana, 2015). Hemeproteins are involved in a variety of crucial biological processes, such as reversible binding of oxygen to hemoglobin and myoglobin, which are responsible for oxygen transport and storage, respectively; transport of electrons in oxidative phosphorylation process through cytochromes; oxygen metabolism through the enzymes oxydase, peroxydases, catalase and hydrolase (Ana, 2015). 
The physiology of iron trafficking and metabolism has been well evaluated over the last 20 years, and several comprehensive reviews have been published on the subject (Fuqua et al., 2012). Many proteins have been identified playing roles in iron metabolism. Some proteins such as ferritin or Tf are the main cargos of blood iron, whereas peptides such as iron regulatory proteins (IRPs), hepcidin, and matriptase (Mt2) are key determinants of iron regulation at different physiological levels. A set of different proteins, notably divalent metal transporter-1 (DMT1), ferroportin (FPN1), and transferrin receptors (Tfrs) in association with ferroxidases such as duodenal cytochrome B, ceruloplamin $(\mathrm{Cp})$ and heme carrier protein (HCP1), are involved in the cellular membrane transportation of iron (Sophie et al., 2014). Others proteins such as myoglobin $(\mathrm{Mb}), \mathrm{Hb}$, and many different enzymes are the 'end' products of iron metabolism, because they require iron for their functions. Transferring is a monomeric glycoprotein produced in the liver and consists of a polypeptide chain of 679 amino acids in two homologue domains, Nterminal and $\mathrm{C}$ - terminal, each containing a $\mathrm{Fe}+{ }^{3}$ binding site. Transferring has three main functions (Ana, 2015):

a) $\mathrm{Fe}+3$ solubilization

b) High-affinity iron binding avoiding that the free metal produces free radicals and

c) Iron supply to cells, involving its interaction with membrane receptors, that is, transferring receptors

Throughout life, humans maintain a constant concentration of iron ranging from 35 to 45 $\mathrm{mg} / \mathrm{Kg}$. These levels are maintained by a positive iron balance and equilibrium between absorption and loss. The average intake of normal individuals is approximately $12-18 \mathrm{mg}$ of iron/day and 1-2 $\mathrm{mg}$ are absorbed. The daily iron excretion in case of no bleeding is roughly one milligram, and it occurs mainly through the gastrointestinal tract by exfoliation of epithelial cells, bile secretion, and epidermal scaling, and also by the urinary tract. In the reproductive phase, women loose, in average, $2 \mathrm{mg}$ of iron/day, due to menses (Ana, 2015). The absorption of iron is a regulated physiological process that serves as a primary mechanism to control iron balance. The most important stimuli for iron absorption are tissue iron storage, rate of erithropoesis and tissue hypoxia. Absorption increases response to tissue hypoxia and production of erythrocytes.Most iron in diet is in the ferric form. This form is soluble in acid $\mathrm{pH}$ and insoluble in alkaline $\mathrm{pH}$, forming insoluble polyhydroxy groups that make iron biologically unavailable. In the stomach, iron is mobilized from food by chelation of mucins, ascorbate and histidine, among others. Hemeprotein iron is absorbed from food (meats) in a different and more efficient manner than inorganic iron. Heme-iron enters in the intestinal mucosa cells as an intact metalloporphyrin, after globin release by proteolitic enzymes. This entry is facilitated by heme receptors and a vesicular transport system. In the cell, $\mathrm{Fe}+2$ is released from porphyrin by microssomalheme-oxygenase and enters into circulation like the inorganic form (Conrad, 2002).

\section{PATHOPHysiology OF HyPERTENSiON}

Hypertension is a chronic elevation of blood pressure that, in the long-term, causes end-organ damage and results in increased morbidity and mortality. Blood pressure is the product of cardiac output and systemic vascular resistance. It follows that patients with arterial hypertension may have an increase in cardiac output, an increase in systemic vascular resistance, or both. In the younger age group, the cardiac output is often elevated, while in older patients increased systemic vascular resistance and increased stiffness of the vasculature play a dominant role. Vascular tone maybe elevated because of increased a-adrenoceptor stimulation or increased release of peptides such as angiotensin or endo the lins (Foe"x and Sear, 2015). The final pathway is an increase in cytosolic calcium in vascular smooth muscle causing vasoconstriction. Several growth factors, including angiotensin and endothelins, because an increase in vascular smooth muscle mass termed vascular remodelling. Both an increase in systemic vascular resistance and an increase in vascular stiffness augment the load imposed on the left ventricle; this induces left ventricular hypertrophy and left ventricular diastolic dysfunction (Foe“x and Sear, 2015).

The autonomic nervous system plays an important role in the control of blood pressure. In hypertensive patients, both increased release of, and enhanced peripheral sensitivity to, norepinephrine can be found. In addition, there is increased responsiveness to stressful stimuli. Another feature of arterial hypertension is a resetting of the baroreflexes and decreased baroreceptor sensitivity. The renin-angiotensin system is involved at least in some forms of hypertension (e.g.renovascular hypertension) 
and is suppressed in the presence of primary hyper aldosteronism. Elderly or black patients tend to have low-renin hypertension. Others have high-renin hypertension and these are more likely to develop myocardial infarction and other cardiovascular complications. In human essential hypertension, and experimental hypertension, volume regulation and the relationship between blood pressure and sodium excretion (pressure natriuresis) are abnormal. Considerable evidence indicates that resetting of pressure natriuresis plays a key role in causing hypertension. In patients with essential hypertension, resetting of pressure natriuresis is characterized either by a parallel shift to higher blood pressures and salt-insensitive hypertension, or by a decreased slope of pressure natriuresis and salt-sensitive hypertension (Foe" $\mathrm{x}$ and Sear, 2015).

\section{Pulmonary Hypertension}

Pulmonary hypertension encompasses a group of conditions characterized by raised blood pressure in the pulmonary arteries. The formal diagnosis requires right heart catheterization: $\mathrm{PH}$ is defined as a mean pulmonary arterial pressure $\geq 25 \mathrm{mmHg}$ at rest. There is a further hemodynamic division into pre- and postcapillary $\mathrm{PH}$ depending on whether the pulmonary artery wedge pressure (a measure of left atrial pressure) is $\leq 15 \mathrm{mmHg}$ (pre-capillary $\mathrm{PH}$ ) or $>15 \mathrm{mmHg}$ (post-capillary $\mathrm{PH}$ ). $\mathrm{PH}$ is divided into five clinical groups, each group of which shares similar pathophysiology and anticipated response to treatment (Galieet al., 2016).

Group $1 \mathrm{PH}$ is known as pulmonary arterial hypertension (PAH). All the conditions within this group have pre-capillary hemodynamics, a raised pulmonary vascular resistance (PVR) and no evidence of significant lung disease (Group 3 $\mathrm{PH}$ ) or thromboembolic disease (Group 4). The main disorders presenting with $\mathrm{PAH}$ are congenital heart disease (predominantly Eisenmenger Syndrome), scleroderma associated PAH and idiopathic (i)PAH (a diagnosis of exclusion) (Lathaet al., 2018). Within the clinical phenotype of Group $1 \mathrm{PAH}$, a small proportion will have a family history and most will carry a genetic abnormality in one of the genes associated with the condition, predominantly, bone morphogenetic protein receptor (BMPR) 2 (Soubrier et al., 2013). All members of Group 1 have similar histology with remodeling of pulmonary arterioles (diameter < $500 \mu \mathrm{M})$. This involves hyperplasia of cells encompassing all three layers of the vessel wall, although predominantly smooth muscle. The resulting increase in PVR increases the afterload on the right ventricle (RV) provoking $\mathrm{RV}$ hypertrophy, enlargement and eventually failure (Tuderet al., 2013). PAH is always associated with increased morbidity and mortality, but with some heterogeneity depending on sub-type. Patients with iPAH have one of the worse prognoses with a pre-treatment era median survival of only 2.8 years, comparable to many advanced cancers. In addition, it has a female predominance and tends to affect younger adults (Lathaet al., 2018).

On the other, in heritable PAH, defects predominantly in the BMPR2 gene increase susceptibility to developing PAH. However, the penetrance of the genetic defect remains low, suggesting other "hits" are necessary. Additional insults are likely to include: infection, exposure to hypoxia, exposure to serotoninergic drugs and pregnancy related changes in female hormone levels (Tuder et al., 2013). Although not proven, one of the earliest abnormalities is probably endothelial cell dysfunction leading to an imbalance of vasoactive molecules: increased production of the vasoconstrictor and mitogen, endothelin (ET)-1 and reduced production of nitric oxide (NO) and prostacyclin (PGI2), both vasodilators and anti-proliferative agents (Latha et al., 2018). Damage to the endothelium may expose the underlying smooth muscle (SM) to cytokines and serum factors that promote proliferation. As the disease progresses, it is likely that apoptotic resistant endothelial cells lead to neointimal formation and fibrosis and the formation of plexiform lesions. The adventitia is also involved with increased numbers of fibroblasts and extra-cellular matrix protein deposition. Inflammatory changes are often observed surrounding remodeled vessels, although the exact contribution of inflammation (marker or mediator) remains unclear. However, circulating cytokines, such as IL-6, IL-8, and IL-10 are increased in patients with PAH and correlate with outcome (Latha et al., 2018).

Nitric oxide (NO) mediates the vasodilatation produced by acetylcholine, bradykinin, sodium nitroprusside and nitrates. In hypertensive patients, endothelial-derived relaxation is inhibited. The endothelium synthesizes endothelins, the most powerful vasoconstrictors. The generation of, or sensitivity to, endothelin-1 is no greater in hypertensive than it is in normotensive subjects. Nonetheless, the deleterious vascular effects of endogenous, 
endothelin-1 may be accentuated by reduced generation of nitric oxide caused by hypertensive endothelial dysfunction (Foe $\mathrm{x}$ and Sear, 2015).

\section{EFFECTS OF IRON DEFICIENCY ON HEART FUNCTION}

In the heart, iron is present predominantly in ferritin and within the mitochondrial compartment. The latter is in the form of ironsulphur clusters and haeme functional groups. Iron in iron-sulphur and heme groups is required for electron transfer and oxygen activation in oxidative phosphorylation (Samira, 2019). Additionally, labile-free iron is required for oxygen activation by dioxygenases, and as a catalyst for the production of reactive oxygen species (ROS) which are essential for redox signalling (Burgoyne et al., 2012).

Anaemia indirectly affects cardiac function by reducing muscle exercise capacity, and directly by limiting oxygen availability for use in oxidative phosphorylation within cardiomyocytes. As well as limiting iron availability to the bone marrow, iron deficiency also limits the iron that is available for uptake by non-erythroid tissues. Recently, the direct effects of limited iron availability in cardiomyocytes were demonstrated in a series of experimental models. These models were designed to generate iron deficiency in the heart while maintaining intact systemic iron homeostasis. In a mouse model lacking cardiomyocytetransferrin receptor 1 (TfR1), iron levels in the heart were severely reduced, resulting in fatal heart failure in the second week of age, in part, due to failure of mitochondrial respiration (Xu et al., 2015). Another mouse model of cardiac iron deficiency, achieved through increased iron export in cardiomyocytes, also resulted in heart failure, likely explained by a decrease in the activity of the electron transport chain. Studies of dietary iron restriction in animal models have also shown reduced cardiac contractility due to impaired calcium handling in the iron-deficient hearts (Chung et al., 2019). The effects of iron deficiency on calcium handling recapitulated the changes induced by hypoxia in cultured cardiomyocytes, supporting the notion that they are driven by reduced oxygen delivery in anemic animals (Chung et al., 2019). However, the direct effects of iron deficiency on hypoxic signaling in the heart could also be important in this context. Indeed, oxygen sensing by hypoxia-inducible factor (HIF) prolyl hydroxylase (PHD) is modified by intracellular iron availability, due to the requirement of PHDs for iron as a co-factor. In summary, iron deficiency affects heart function through anaemia-dependent and anaemia-independent mechanisms.

\section{Treating Iron Deficiency in Heart FAILURE}

The beneficial effects of intravenous iron supplementation in patients with chronic heart failure likely depend on a combination of mechanisms, including an improvement in exercise capacity and oxygen delivery to tissues. Animal studies have also shown that such interventions can directly correct cardiac iron deficiency (Samira, 2019). Myocardial iron content has been shown to be reduced in patients with heart failure, where it is shown to be associated with reduced activity of the citric acid cycle enzymes aconitase and citrate synthase (Samira, 2019). Thus, correction of myocardial iron deficiency may well constitute an additional mechanism through which iron interventions improve outcomes in CHF.In this context, avoidance of iron toxicity in the heart should be an important consideration. Indeed, the heart's function is particularly sensitive to excess iron accumulation, as demonstrated by the iron overload cardiomyopathy that occurs in patients with iron overload conditions such as hemochromatosis and thalassemia (Samira, 2019). In line with this, a mouse model harbouring a cardiomyocyte-specific deletion of the iron export protein ferroportin (FPN) was also found to develop fatal cardiomyopathy as a consequence of cardiomyocyte iron-overload (Haddad et al., 2017)..

An alternative approach to supplementing iron to the heart is to target the molecular machinery of cellular iron homeostasis in cardiomyocytes. Like other cells in the body, cardiomyocytesutilise the iron regulatory proteins (IRPs) to orchestrate iron uptake, utilisation, and storage. Mice with the cardiacspecific deletion of IRPs fail to increase LV systolic function in response to the dobutamine challenge and have worse LV dysfunction and higher mortality following myocardial infarction (Haddad et al., 2017). At the same time, IRP activity has been shown to be reduced in the failing human heart (Haddad et al., 2017). Together, these findings support the notion that myocardial iron deficiency may develop as a consequence of reduced IRP activity in the failing heart, and that this, in turn, impairs 
cardiac reserve and response to injury. Enhancement of the IRP pathway, by increasing the expression or stability of IRPs, may, therefore, present novel therapeutic strategies in heart failure. The advantage of such an approach over systemically administered iron compounds is that it may enable a more measured correction of intracellular iron levels within cardiomyocytes, reducing the risk of iron toxicity. Recently, another mechanism of cellular iron homeostasis was identified, in which cardiomyocytesutilisehepcidin, also known as human antimicrobial peptide (HAMP), in an autocrine manner to regulate the iron export protein FPN. Despite maintenance of normal systemic iron levels, deletion of hepcidin specifically in cardiomyocytes resulted in fatal LV failure in mice .Cardiomyocyte-specific knock-in of the FPN isoform C326Y, which retains its iron export function but loses its hepcidin binding, also resulted in heart failure of a similar nature and time-course to that seen in animals lacking cardiomyocytehepcidin deficiency (Lakhal-Littleton et al., 2015). In both mouse models, increased FPN-mediated iron export caused the depletion of iron from cardiomyocytes. Heart failure in cardiac hepcidin-knockouts could be prevented by intravenous iron supplementation. Additionally, animals with hepcidin-deficient hearts developed greater hypertrophic response to sustained dietary iron restriction than their littermate controls, indicating that the cardiac hepcidin/FPN axis functions to protect the heart in the setting of systemic iron deficiency (Lakhal-Littleton et al., 2015). These studies identify the cardiac hepcidin/FPN axis as a nonredundant component of cellular iron homeostasis in cardiomyocytes, and further show that the control of the intracellular iron pool in these cells is required for normal cardiac function deficiency (Lakhal-Littleton et al., 2015). Studies comparing mice harbouring tissue-specific with those harbouring a ubiquitous disruption of the hepcidin/FPN axis support the notion that intracellular iron levels within cardiovascular cells are the sum of fluxes through both the systemic and the local hepcidin/FPN axes.

\section{LABORATORY DIAGNOSIS OF IRON}

The measurement of haematologic indicators in general and haemoglobin in particular is widely available in clinical and research laboratories by flow cytometry on fully automated cell counters. These instruments can count different blood cells with good precision and high sample throughput. Battery-operated, hand-held haemoglobinometers can provide on-the-spot results from a single drop of blood collected in the field. However, these instruments require freshly collected blood, and if capillary blood is used, the proper sampling technique is critical to obtaining a valid specimen (Christine and Anne, 2017). The measurement of serum-based biochemical indicators is generally carried out on fully-automated clinical analyzers available in many laboratories. Protein-based indicators such as SF, transferrin, and soluble transferrin sTfR are measured by immunoassays such as ELISA, whereas serum iron, TIBC, and UIBC are measured on chemistry and auto analyzers by using a colorimetric reaction with ferrine or ferrozine as a chromogen to form a color complex with iron. Most clinical analyzers measure UIBC because it is more easily automated than Total iron Biding Capacity (TIBC). The measurement precision of UIBC is good at high concentrations as found in iron depletion but worse at low concentrations in the presence of iron overload. Red cell counts, haemoglobin, mean corpuscular volume (MCV) hemoglobin concentration (MCHC), and red cell distribution width (RDW) can be used to determine the iron level (Salou et al., 2019).

Treatments: Summary of Clinical EVIDENCE WITH IRON REPLACEMENT IN PAH

There are very few clinical trials investigating the effect of iron replacement in patients with PAH. (Viethenet al., 2014) presented the results of 20 iron deficient patients with mixed PAH etiology, given IV ferric carboxymaltose $(1,000$ $\mathrm{mg}$ ) in an open label fashion. Compared to a non-treated group, there was an improvement in iron status, significant increase in 6-min walk test and quality of life score (Viethenet al., 2014). There have been two trials in specific PAH sub-types, described below.

\section{IRON THERAPY IN CYANOTICCON GENITALHEARTDISEASE}

There are no randomized, placebo controlled studies investigating the effect of iron replacement in the cyanotic congenital heart disease population. However, (Tay et al., 2011) studied 25 iron-deficient cyanotic CHD patients, 14 of which had Eisenmenger Syndrome, who received oral iron replacement in a prospective open label manner (Tay et al., 2011). Oral ferrous fumarate was titrated to a maximum of $200 \mathrm{mg}$ tds. After 3 months of treatment, 
hemoglobin, ferritin and transferrin saturation had all significantly increased. Significant improvements were also seen in quality of life and 6MWT, although peak VO2 was unchanged. Oral treatment was well tolerated with no complications. In clinical practice in the cyanotic CHD population treatment of iron deficiency (as a complication of secondary erythrocytosis) is managed quite aggressively, usually with intravenous iron replacement. In 142 consecutive cyanotic CHD patients of which $49 \%$ were ES, treatment with IV ferrous carboxymaltose $\quad(500 \mathrm{mg}$; FerrinjectTM $)$ resulted in improvement in hemoglobin, hematocrit, ferritin, and transferrin saturation. There were no cases of excessive erythrocytosis with very few complications (personal communication).

\section{IRON THERAPY AND IDIOPATHIC Pulmonary Arterialhy Pertension}

There is only one published study on the use of iron replacement in patients with iPAH (Gerrina et al., 2015). Fifteen patients with iPAH and iron deficiency received a single, high dose of IV iron in an open label fashion. After 12 weeks, the primary endpoint, 6MWT, was not changed. However, exercise endurance time and aerobic capacity increased significantly without change in cardiac function. There was improved quadriceps muscle oxygen handling. IV iron was well tolerated and there was an improvement in quality of life. A randomized, placebo controlled trial is currently active and the results are eagerly awaited (Howard et al., 2013).

\section{Iron THERAPY IN OTHER FORMS OF PH}

There are no other studies investigating the use of iron replacement in other forms of $\mathrm{PH}$ apart from experimental studies in acute hypoxia driven PH. (Bart et al., 2016)investigated the effect of $1 \mathrm{~g}$ IV iron (orsaline) on increases in PASP associated with $6 \mathrm{~h}$ of hypoxia in 22 subjects. Patients receiving iron before hypoxia had a 50\% reduction in PASP rise which was sustained up to 43days after iron therapy (Bart et al., 2016).

\section{Changes in Iron Status in Hypertensive Patients}

There is evidence demonstrating the multifactorial nature for the development of iron deficiency in pulmonary hypertension. The mechanisms involved are a reduction in iron absorption caused by gastrointestinal edema associated with systemic venous congestion, increased iron consumption due to secondary polycythaemia and chronic hypoxemia (Rodrigo and Tomas, 2019), blood loss as a consequence of chronic anticoagulation and abnormal hepcidin serum levels.

Similarly, another study in chronic thromboembolic pulmonary hypertension patients found no difference in iron parameters between patients with chronic thromboembolic pulmonary hypertension and controls (Jankowich et al., 2016). Studies have linked increased serum ferritin levels in non-pathologic conditions, reflecting subclinical iron overload, to an increased risk of hypertension (MeeKyoung et al., 2011). There are three possible mechanisms to explain this association. First, insulin resistance could be the main mechanism involved, as numerous studies support a link between insulin resistance and hypertension. According (MeeKyoung et al., 2011), ferritin levels were associated with insulin resistance and the development of type 2 diabetes.5 Second, this association can be mediated by the presence of fatty liver disease. This is associated with a progressive increase in BP over time and with incident hypertension. Iron overload might play a role in the pathogenesis of fatty liver disease by increasing insulin resistance and oxidative stress (MeeKyoung et al., 2011). Total iron biding capacity (TIBC) levels display variations in disorders of iron metabolism and are decreased in chronic inflammatory disorders and malignancies (Naveed et al., 2016). The level of serum ferritin can change dramatically before and after menopause. Ferritin levels are generally low in women of childbearing age due to iron lost during menstruation, and then the levels increase after menopause. In addition, serum ferritin concentrations in women are difficult to study because they are affected by many variables such as pregnancy, hormone therapy, and gynaecological diseases (DongHoon et al., 2018). A high iron load can also be reflected in high ferritin levels, but by a decreased TIBC. Although negatively correlated, both ferritin and TIBC were positively associated with the development of hypertension

\section{Risk MANAgEMENT OF HyPERTENSION}

As well as pharmacological measures for the control of blood pressure, there should be active treatment of those factors known to increase the risk of hypertension. There are two distinct 
measures. First, those that lower blood pressure, for example weight reduction, reduced salt intake, limitation of alcohol consumption, physical exercise, increased fruit and vegetable consumption, and reduced total and saturated fat intake. Second, those that that reduce cardiovascular risk, for example stopping smoking; replacing saturated with polyunsaturated and monounsaturated fats; increased oily fish consumption; and reduced total fat intake. Because hypertensive patients are at very high risk of coronary artery disease, other therapeutic measures include aspirin and statin therapies (Foe"x and Sear, 2015). Losedose aspirin is effective in the prevention of thrombotic events such as stroke and myocardial infarction; this is also true in hypertensive patients whose blood pressure is well controlled. The risk of severe bleeding is very low provided blood pressure is reduced to below $150 / 90 \mathrm{~mm}$ Hg. The benefits of lipid-lowering drug treatment with statins are well established in coronary heart disease and in cerebrovascular disease, two conditions frequently associated with arterial hypertension (Foe" $\mathrm{x}$ and Sear, 2015).

\section{CONCLUSION}

Iron is a key player in haemoglobin synthesis and erythrocyte production. At the same time, it is a potent poison to mammalian cells and an indispensable nutrient. Therefore, its metabolism in mammalians is very complex and stringently controlled by many different genes and proteins. Restoring iron levels improves quality of life as it is an essential nutrient for survival.

\section{REFERENCES}

[1] Ana, M., (2015). Iron metabolism. Reviewing Basic Sciences.3(2):123-126.

[2] Bart, N.K., Curtis, M.K., Cheng, H.-Y., Hungerford,S.L., Mclaren, R., and Petousi, N., (2016). Elevation of iron storage in humans attenuates the pulmonary vascular response to hypoxia. Journal of Applied.Physiology.121, 537-544.

[3] Burgoyne, J.R., Mongue-Din, H., Eaton, P., and Shah, A.M. ( 2012) Redox signaling in cardiac physiology and pathology. Circulatory. Research.111, 1091-1106.

[4] Chung, Y.J., Luo, A., Park, K.C., Loonat, A.A., Lakhal-Littleton, S., Robbins, P.A., and Swietach, P. (2019). Iron-deficiency anemia reduces cardiac contraction by downregulating RyR2 channels and suppressing SERCA pump activity. JCI Insight.4, 125618.
[5] Conrad, M.E., Umbreit J.N. (2002) Pathways of iron absorption. Blood Cells Molecules and Diseases, 29(3):336-55.

[6] Cotroneo, E., Ashek, A., Wang, L., Wharton, J., Dubois, O., and Bozorgi, S., (2015). Iron homeostasis and pulmonary hypertension: iron deficiecy leads to pulmonary vascular remodeling in the rat. Circulation research. 116(10): 1680-90.

[7] Foe"x, P., and Sear, J.W. (2015). Hypertension: pathophysiology and treatment. Continuing Education in Anaesthesia, Critical Care \& Pain. 4 (3)71-75.

[8] Fuqua, B.K., Vulpe, C.D., and Anderson, G.J. (2012).Intestinal iron absorption. Journal of Trace Elements in Medicine and Biology. 26:115-119.

[9] Galie, N., Humbert, M., Vachiery, J. L., Gibbs, S., Lang, I., and Torbicki, A. (2016). ESC/ERS guidelines for the diagnosis and treatment of pulmonary hypertension: the joint task force for the diagnosis and treatment of pulmonary hypertension of the European society of cardiology (ESC) and the European respiratory society (ERS): endorsed by: association for European paediatric and congenital cardiology (AEPC), international society for heart and lung transplantation (ISHLT). European Heart Journal.37 67-119.

[10] Haddad, S., Wang, Y., Galy, B., KorfKlingebiel, M., Hirsch, V., Baru, A.M., Rostami, F., Reboll, M.R., Heineke, J., and Flögel, U. (2017). Iron-regulatory proteins secure iron availability in cardiomyocytes to prevent heart failure. European Heart Journal. 2017, 38, 362-372.

[11] Jankowich, M., Elston, B., Evans, S.K., Wu, WC.,Choudhary, G. (2016) Relationship of Iron Deficiency and Serum Ferritin Levels with Pulmonary Hypertension: The Jackson Heart Study. PLOS ONE11(12): e0167987.

[12] Lakhal-Littleton, S., Wolna, M., Chung, Y.J., Christian, H.C., Heather, L.C., Brescia, M., Ball, V., Diaz, R., Santos, A., and Biggs, D. (2015). An essential cell-autonomous role for hepcidin in cardiac iron homeostasis. eLife. $\mathbf{5}$, e19804.

[13] Latha, R., Sofia, L., Pedersen, Q. K. Toe, G. J., Quinlan, and Stephen J. (2018). Pulmonary Arterial Hypertension: Iron Matters. Frontiers in Physiology.9:641.

[14] Matthew, J., Beth, E., Samuel, K., Evans, S.K., Wen-Chih, W., and Gaurav, C., (2016). Relationship of Iron Deficiency and Serum Ferritin Levels with Pulmonary Hypertension: The Jackson Heart Study. PLoS ONE.11(12): e0167987.

[15] MeeKyoung, Kim., Ki Hyun, Baek,, Ki-Ho, S., Moo, Kang., JiHoon, C., JiCheol B., Cheol, Y. P., Won Y. L., and Ki W. (2012) . Increased 
Serum Ferritin Predicts the Development of Hypertension Among Middle-Aged Men. American Journal of Hypertension 25(4); 493496.

[16] Naveed, A., Aamir I., Tariq R., Zujaja, H. H., Saima, B., and Muhammad A. (2016). Diagnostic Accuracy of Serum Iron and Total Iron Binding Capacity (TIBC) in Iron Deficiency.Journal of the College of Physicians and Surgeons Pakistan26(12): 958961.

[17] Nickol, A.H., Frise, M.C., Cheng, H.Y., McGahey, A., McFadyen B.M., Harris-Wright, T., Bart, N.K., Curtis, M.K., Khandwala, S., and O'Neill, D.P. (2015). A cross-sectional study of the prevalence and associations of iron deficiency in a cohort of patients with chronic obstructive pulmonary disease. BMJ Open, 5, e007911.

[18] Rhodes, C.J., Howard, L.S., Busbridge, M., Ashby, D., Kondili, E., and Gibbs, J.S. (2011). Iron deficiency and raised hepcidin in idiopathic pulmonary arterial hypertension: clinical prevalence, outcomes, and mechanistic insights. Journal of the American College of Cardiology.58(3):300-9.

[19] Ruiter, G., Lankhorst, S., Boonstra, A., Postmus, P.E., Zweegman, S., Westerhof, N., van der Laarse, W.J., and Vonk-Noordegraaf,
A. (2011). Iron deficiency is common in idiopathic pulmonary arterial hypertension. European Respiratory Journal.37:1386-1391.

[20] Samira, L., (2019). Iron Deficiency as a Therapeutic Target in Cardiovascular Disease. Pharmaceuticals. 12(125)2-9.

[21] Sophie, W. A., Gérard, W., Christoph, G., Andreas, B., Beat, M. F., Bernard, F., and Jean T. (2014). Physiology of Iron Metabolism. Transfussion Medicine and Hemotherapy. 41:000-000.

[22] Soubrier, F., Chung, W. K., Machado, R., Grünig, E., Aldred, M., and Geraci, M. (2013). Genetics and Genomics of Pulmonary Arterial Hypertension. Journal of American Collection of Cardiology.62 D13-D21.

[23] Viethen, T., Gerhardt, F., Dumitrescu, D., Knoop-Busch, S., Ten Freyhaus, H., and Rudolph,T.K. (2014). Ferric carboxymaltose improves exercise capacity and quality of life in patients with pulmonary arterial hypertension and iron deficiency: a pilot study. International Journal of Cardiology.175, 233-239.

[24] Xu, W., Barrientos, T., Mao, L., Rockman, H.A., Sauve, A.A., and Andrews, N.C. (2015) Lethal Cardiomyopathy in Mice Lacking Transferrin Receptor in the Heart.Cell Report. 13, 533-545.

Citation: Obeagu Emmanuel Ifeanyi, "Changes in Iron Status of Hypertensive Patients", International Journal of Research Studies in Medical and Health Sciences. 2020; 5(2): 06-14.

Copyright: (C) 2020 Obeagu Emmanuel Ifeanyi, This is an open-access article distributed under the terms of the Creative Commons Attribution License, which permits unrestricted use, distribution, and reproduction in any medium, provided the original author and source are credited. 